\title{
A REACTION-DIFFUSION EQUATION WITH MEMORY
}

\author{
Maurizio Grasselli and Vittorino Pata \\ Dipartimento di Matematica "F.Brioschi" \\ Politecnico di Milano \\ Via Bonardi 9, I-20133 Milano, Italy
}

\begin{abstract}
We consider a one-dimensional reaction-diffusion type equation with memory, originally proposed by W.E. Olmstead et al. to model the velocity $u$ of certain viscoelastic fluids. More precisely, the usual diffusion term $u_{x x}$ is replaced by a convolution integral of the form $\int_{0}^{\infty} k(s) u_{x x}(t-s) d s$, whereas the reaction term is the derivative of a double-well potential. We first reformulate the equation, endowed with homogeneous Dirichlet boundary conditions, by introducing the integrated past history of $u$. Then we replace $k$ with a timerescaled kernel $k_{\varepsilon}$, where $\varepsilon>0$ is the relaxation time. The obtained initial and boundary value problem generates a strongly continuous semigroup $S_{\varepsilon}(t)$ on a suitable phase-space. The main result of this work is the existence of the global attractor for $S_{\varepsilon}(t)$, provided that $\varepsilon$ is small enough.
\end{abstract}

1. Introduction. Let $(0, \ell) \subset \mathbb{R}$ be a bounded interval. For $u=u(x, t):(0, \ell) \times$ $\mathbb{R} \rightarrow \mathbb{R}$, we consider the equation

$$
u_{t}-\int_{0}^{\infty} k(s) u_{x x}(t-s) d s+\phi(u)=f, \quad t>0,
$$

where $k:[0, \infty) \rightarrow[0, \infty)$ is a suitable memory kernel, the function $\phi$ is a smooth nonlinearity with (at most) cubic growth, and $f$ is a given external force, which is supposed to be independent of time for the sake of simplicity.

Equation (1.1), endowed with the boundary conditions

$$
u(0, t)=u(\ell, t)=0, \quad t \geq 0,
$$

has been proposed in [14] to model the behavior of certain viscoelastic fluids. There, $u$ represents the velocity of the fluid and $\phi(\xi)=\xi^{3}-R \xi, R$ being the Rayleigh number. The kernel $k$ is assumed to be of Jeffreys type, namely,

$$
k(s)=(1-\sigma) \frac{e^{-\frac{s}{\varepsilon}}}{\varepsilon}+\sigma \delta(s)
$$

where $\varepsilon>0$ is the relaxation time, $\sigma \in[0,1]$ is the ratio of retardation to relaxation times, and $\delta$ is the Dirac mass at zero. Observe that for $\sigma=1$ we recover the

2000 Mathematics Subject Classification. Primary: 35B41; Secondary: 45K05, 76A10.

Key words and phrases. Reaction-diffusion equations, memory effects, global attractors.

This work was partially supported by the Italian MIUR PRIN Research Projects Modellizzazione Matematica ed Analisi dei Problemi a Frontiera Libera and Aspetti Teorici e Applicativi di Equazioni a Derivate Parziali, and by the Italian MIUR FIRB Research Project Analisi di Equazioni a Derivate Parziali, Lineari e Non Lineari: Aspetti Metodologici, Modellistica, Applicazioni. 